\title{
Bayesian analysis of trial-based cost-effectiveness in the presence of missing data: effects of including covariates on efficiency of estimation in the ASTER trial
}

\author{
Linda Sharples $^{1 *}$, Christopher Jackson ${ }^{1}$, Ella Wheaton ${ }^{2}$, Robert Rintoul ${ }^{3}$ \\ From Clinical Trials Methodology Conference 2011 \\ Bristol, UK. 4-5 October 2011
}

\section{Objectives}

Bayesian estimation of missing resource use data and expected costs in the ASTER trial of endosonographic staging followed by surgical staging if negative (ES), compared with surgical staging alone (SS), in candidates for lung cancer surgery.

To assess how covariates, that are included in the model to justify a "missing at random" assumption, affect estimates of expected costs.

\section{Methods}

ASTER was a prospective, international, open-label, randomised-controlled study, with a trial-based economic analysis over 6 months. Due to delays in starting the health economic study, resource use data were collected prospectively for the second half of the study only. Although resource use data could be ascertained retrospectively, some items were difficult to ascertain once patients had been discharged from the trial centre. A Bayesian parametric model was developed to estimate missing resource use items and expected costs. Missing resource use data were modelled using Binomial, Poisson, over-dispersed equivalents of these, or using a hurdle count model if only a proportion of the patients had the event (e.g. chemotherapy). Covariates considered were randomisation group, centre, age, sex and stage of lung cancer. The total expected cost was calculated as the sum of the resource use component-specific expected costs for each randomisation group.

${ }^{1}$ MRC Biostatistics Unit, Cambridge, UK

Full list of author information is available at the end of the article

\section{Results}

ES was more sensitive, resulted in fewer futile thoracotomies and had better utility during staging than SS. All patients had initial diagnostic tests and management recorded but subsequent resource use components were missing for $10-20 \%$ of cases, and only $71 \%$ had complete resource use data. Using the complete cases only, the mean 6 month cost of ES was $£ 10,614(£ 8515, £ 13,073)$ per patient versus $£ 11,788$ ( $£ 9053, £ 15,321)$ for SS, mean difference $£ 1174(-£ 948, £ 3912)$, so that ES was cheaper but with considerable uncertainty in these estimates. The Bayesian model aimed to recapture power lost due to missing data and when randomisation group was the only covariate, point estimates were reduced by $5 \%$ and posterior standard deviations were reduced by $10 \%$ compared with complete case analysis. Inclusion of other covariates resulted in small subgroups, imprecise point estimates for covariates and a resulting increase in the posterior variance for expected costs.

\section{Conclusions}

Resource use in patient groups is highly variable and trials are rarely powered for secondary outcomes that drive costs, so that inclusion of many parameters in a Bayesian analysis may result in inefficient estimation. Covariate selection should consider both the missing data mechanism and efficiency.

\section{Acknowledgements}

We acknowledge to contribution of the ASTER trial investigators. The UK data collection and cost-effectiveness study were funded by the National Institute for Health Research Health Technology Assessment programme. 


\section{Author details}

${ }^{1}$ MRC Biostatistics Unit, Cambridge, UK. ${ }^{2}$ Cancer Research UK, London, UK.

${ }^{3}$ Papworth Hospital NHS Foundation Trust, Cambridge, UK.

Published: 13 December 2011

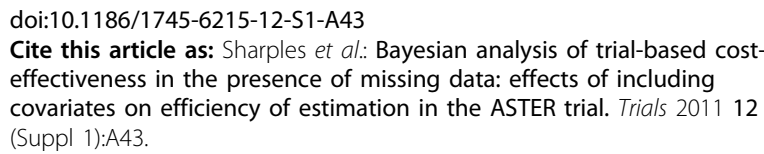

Submit your next manuscript to BioMed Central and take full advantage of:

- Convenient online submission

- Thorough peer review

- No space constraints or color figure charges

- Immediate publication on acceptance

- Inclusion in PubMed, CAS, Scopus and Google Scholar

- Research which is freely available for redistribution 